\title{
The Combination of Surgery and Imatinib in GIST: A Reality for Localized Tumors at High Risk, an Open Issue for Metastatic Ones
}

\author{
Alessandro Gronchi, MD ${ }^{1}$ and Chandrajit P. Raut, MD, Msc ${ }^{2,3,4}$ \\ ${ }^{1}$ Department of Surgery, Sarcoma Service, Fondazione IRCCS Istituto Nazionale Tumori, Milan, Italy; ${ }^{2}$ Department of \\ Surgery, Brigham and Women's Hospital, Boston, MA; ${ }^{3}$ Center for Sarcoma and Bone Oncology, Dana-Farber Cancer \\ Institute, Boston, MA; ${ }^{4}$ Harvard Medical School, Boston, MA
}

Wang et al. update the results of a phase II study on neoadjuvant/adjuvant imatinib mesylate for localized primary and metastatic/recurrent operable gastrointestinal stromal tumors (GIST) with longer follow-up. ${ }^{1}$

Although the term neoadjuvant correctly applies to a therapy provided in the preoperative setting in patients with primary disease, it is highly misleading when applied to a therapy provided before surgery in patients with metastatic disease. In the former setting, in patients with localized disease, the primary therapy is surgery; imatinib plays an adjuvant/neoadjuvant role. In metastatic disease, imatinib is the primary therapy and should not be discontinued; surgery may play an adjuvant role, although this is not yet proven. Indeed, these two scenarios are quite different. They were separately analyzed and should be separately discussed and put in perspective.

\section{PRIMARY DISEASE}

Surgery is the only potentially curative therapy for patients with localized primary GIST. All patients with tumors $>2 \mathrm{~cm}$ in size should undergo surgical resection. ${ }^{2}$ Imatinib should be provided as an adjuvant treatment in patients with a high risk of recurrence. ${ }^{3}$ Adjuvant therapy should be administered for at least 3 years on the basis of the results of the SSGXVIII-AIO phase III trial. ${ }^{4}$ This recently reported trial demonstrated improved recurrence-

(C) Society of Surgical Oncology 2011

Published Online: 30 December 2011

A. Gronchi, MD

e-mail: alessandro.gronchi@ istitutotumori.mi.it free survival and, more importantly, overall survival in patients treated with 3 years of postoperative imatinib compared to those treated with 1 year of therapy.

These results expand on the previously published results of the American College of Surgery Oncology Group (ACOSOG) Z9001 phase III trial, which demonstrated that 1 year of adjuvant imatinib improved progression-free survival compared to placebo but failed to demonstrate an overall survival benefit with short follow-up. ${ }^{5}$

In the ACOSOG trial, the benefit applied to patients with tumors carrying sensitive mutations (KIT exon 11, PDGFRA non-D842V) but did not apply to those with tumors carrying mutations generally unresponsive to lower-dose imatinib (KIT exon 9), insensitive to imatinib altogether (PDGFRA exon 18 D842V, KIT exons 13 and 17, SDHB), or with no identifiable mutations at all (socalled wild type). ${ }^{6}$ It is therefore tempting to speculate that the same apply to the survival benefit shown by SSGXVIIIAIO trial.

In the study by Wang et al., the investigators used a dose of $600 \mathrm{mg} /$ day (because the optimal dose had not yet been defined when the trial was launched). It should be emphasized that this is not the current recommended dose for any of the above mutations. Sensitive mutations should be treated with imatinib $400 \mathrm{mg} /$ day. ${ }^{7}$ Increasing the dose does not influence the type or the chance of response. The optimal dose for patients with KIT exon 9 mutant primary GISTs is unknown in the adjuvant setting, though in the metastatic setting it is imatinib $800 \mathrm{mg} / \mathrm{day} .{ }^{8}$ It is reasonable to consider this dose for patients with localized disease. ${ }^{2}$ Insensitive mutations should not be treated at all.

Critical questions for the surgical oncologist evaluating a patient with a localized, primary GIST are, when should 
imatinib be considered in the neoadjuvant setting, and for how long should it be continued? Wang et al. demonstrate that neoadjuvant treatment is feasible, but their study does not truly detail the magnitude of benefit gained by preoperative therapy or delineate the optimal length of therapy.

First, the patient should in principle have a favorable mutational status; otherwise the treatment would be in vain, allowing the tumor to continue growing. Most GISTs will respond, but it should not be forgotten that-especially in gastric location - the amount of insensitive mutations in the localized setting is less uncommon than what has previously been reported. ${ }^{9}$ Although we do not absolutely need to know the mutational status in advance, we should be aware that mutation is an issue. Alternatively, if mutation status is not determined before treatment, we may also check response very early by computed tomography, positron emission tomography, or contrast-enhanced ultrasound. ${ }^{10}$ If a radiographic response is detected within a month, then the mutation status is likely favorable, and the treatment may be continued without necessarily pursuing mutation testing. If not, then mutation status should be investigated before continuing the treatment.

Second, the resectability of the tumor and the extent of resection necessary should be considered. Other than presentations of clearly inoperable tumors (which are usually treated with imatinib up front) or symptomatic tumors requiring urgent intervention (hemorrhage, perforation, etc.), there are few reasons today to perform extended procedures (i.e., multivisceral resections or formal organ resections) without first attempting preoperative therapy. ${ }^{11}$ For instance, patients with large GISTs who may require a long midline incision for resection may benefit from neoadjuvant imatinib to downstage the operation, potentially converting an open laparotomy approach to a laparoscopic one. Figure 1 illustrates a patient with a large primary gastric GIST measuring $16 \mathrm{~cm}$ at presentation. After 6 months of neoadjuvant imatinib, the tumor measured $10 \mathrm{~cm}$ and could be resected via a laparoscopic partial gastrectomy. Another typical circumstance in which neoadjuvant therapy may be beneficial is in patients with GISTs arising in the esophagus, gastroesophageal junction, duodenum, or distal rectum. Preoperative treatment may shrink the tumor and allow a more conservative local excision. Figure 2 provides the example of a distal rectal GIST that, after neoadjuvant therapy, could be resected with a perineal excision instead of a formal rectal resection. A transanal approach could have also been an alternative. In general, such patients would normally also undergo postoperative adjuvant treatment because of the expected recurrence risk.

The chance of obtaining a response is high and the benefit for tumor shrinkage obvious, so this approach should always be discussed for patients affected by bulky and/or poorly located disease (esophagus, gastroesophageal junction, duodenum, distal rectum), as well as for those who would be candidates for an adjuvant treatment anyway.

Third, in the study of Wang et al., patients underwent surgery between the second and third month. It is now well known that the preoperative treatment may result in sustained tumor shrinkage if provided for a longer duration. ${ }^{12}$ Surgical resection may then be performed between the 6th

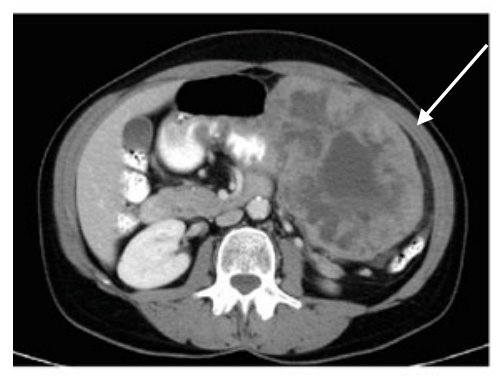

IM $400 \mathrm{mg} /$ day
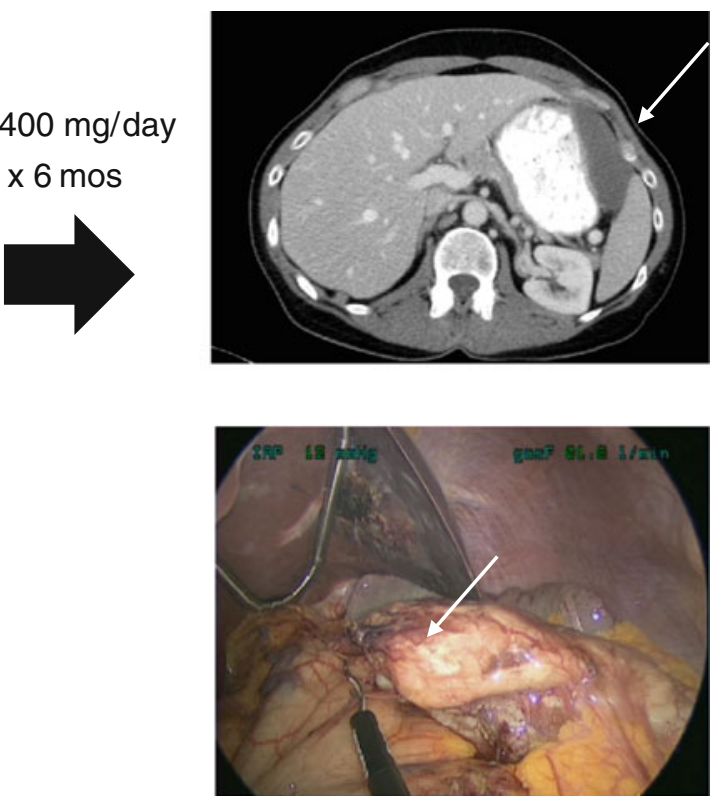

FIG. 1 Contrast-enhanced computed tomographic scan of primary gastric gastrointestinal stromal tumor before (right) and after (left) preoperative imatinib for 6 months. Macroscopic appearance of tumor remnant at laparoscopy, before resection 


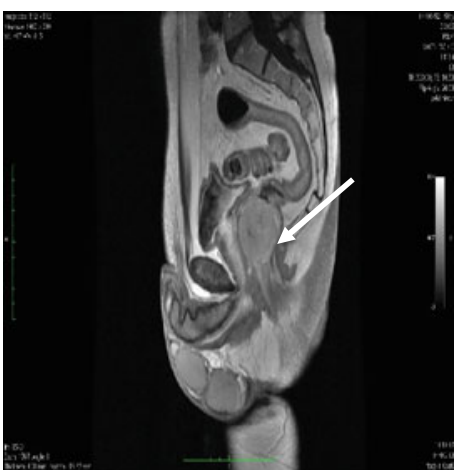

- Local excision through perineal approach
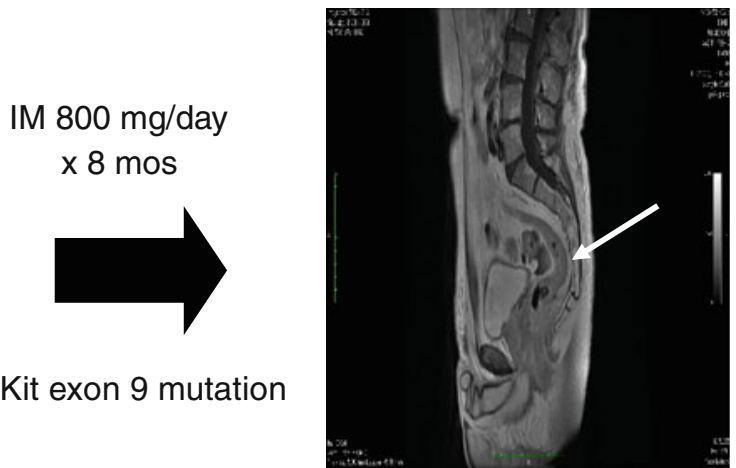

Kit exon 9 mutation
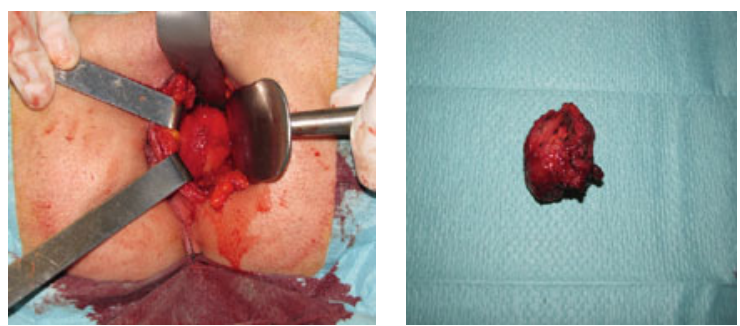

FIG. 2 Contrast-enhanced magnetic resonance image of primary low rectal gastrointestinal stromal tumor before (right) and after (left) preoperative imatinib for 8 months. Because the tumor harbored an exon 9 mutation, the patient was treated with a higher dose. Surgery consisted of local excision rather than a formal low anterior resection and the 12th month, or sooner if the treatment effect plateaus. This allows for optimal tumor shrinkage, or at least shrinkage to the point where there is no further benefit to be gained by further neoadjuvant therapy without the risk of developing secondary resistance. ${ }^{11,12}$ As previously already observed, imatinib can be safely stopped the day before surgery and restarted as soon as postoperative normal feeding is restored. ${ }^{13-19}$

After surgery, patients in the study were allowed to continue therapy for 2 years. The recommendation based on the most current data would be to continue therapy for a total of 3 years. It is not clear, however, whether the 3 years of therapy should be entirely postoperative, or whether it may include the neoadjuvant course. Determining a patient's risk of recurrence after undergoing preoperative therapy is challenging because the mitotic count of the surgical specimen is no longer accurate nor representative of the pretreatment mitotic count. Nevertheless, it is very unlikely that a patient for whom preoperative treatment is considered had a low-risk tumor. Therefore, once we initiate treatment in the preoperative setting, we are committing to treat that patient with imatinib for an additional 3 years.

\section{METASTATIC DISEASE}

Imatinib is the standard first-line treatment for metastatic GIST. The dose-as above-is $400 \mathrm{mg} /$ day except for patients affected by tumors harboring a KIT exon 9 mutation, where $800 \mathrm{mg} /$ day is indicated. ${ }^{7}$

Patients affected by metastatic GIST in the study of Wang et al. were initially scheduled to stop treatment 2 years after surgery. This cannot be recommended today outside of a clinical trial because it is well documented how quickly and inevitably recurrence occurs after imatinib withdrawal in patients with metastatic GIST, even if completely resected. ${ }^{20}$ The investigators in this study appropriately amended the trial to continue indefinite treatment in the recurrent/metastatic cohort. Imatinib is a chronic therapy and should not be interrupted unless unacceptable toxicity and/or disease progression are documented.

The question remains whether to consider surgery in patients whose disease responds to imatinib to potentially prolong its activity. No data are available so far to support this indication. The present study does not really contribute to our understand of this issue. Two trials addressing this question were started in Europe (http://clinicaltrials.gov/ show/NCT00956072) and in China (http://apps.who.int/ trialsearch/trial.aspx?trialid=ChiCTR-TRC-00000244), but both failed to recruit fast enough to meet the target accrual (12 patients were recruited in the European trial and 41 in the Chinese one). This thus remains an open question.

Several single-institutional series consistently reported how feasible and safe it is to operate on these patients. ${ }^{13-19}$ 
Similar results were obtained in patients treated by Wang et al.

The only way to obtain more reliable data on the possible effect of "adjuvant" cytoreductive surgery for patients in response to imatinib is by a prospective observational study. The nonrandomized evidence thus generated would be prospective, contrary to the retrospective nature of published case series analyses on cytoreductive surgery for residual disease in advanced GIST undergoing imatinib treatment and demonstrating ongoing disease stability or response. Of course, there would be a selection bias in the absence of a randomized treatment assignment. Thus, an effort should be made to consider all sources of biases in the study design, and in the analysis and interpretation of results. At each participating center, eligible patients, after being properly informed, could be offered the choice between the two treatment options: surgery plus maintenance therapy with imatinib, or maintenance therapy with imatinib alone. The relative efficacy of the two treatment strategies would be evaluated first by comparing the outcome of patients who chose to undergo surgery versus those who did not (primary analysis), and second by comparing the outcome in centers where the proportion of patients choosing surgery was high (i.e., greater than the overall proportion in all centers combined) versus the outcome in centers where this proportion was low (explanatory analysis).

An effort to build such a study is presently under way and will, we hope, provide data on which to base our future decisions.

A separate question is what to do once limited progression to imatinib is detected. Many experienced centers will consider and offer cytoreductive surgery as salvage therapy in an effort to prolong the time the patient can continue to receive imatinib and postpone the switch to a second-line treatment. Such decisions should be made on a case-by-case basis. ${ }^{2,3}$

\section{CONCLUSIONS}

For patients with localized disease, preoperative treatment with imatinib should always be considered when the expected recurrence risk is not low, the location is difficult, surgery is expected to involve an entire organ or multiple organs, or a more conservative approach could be achieved. Knowing mutational status would be ideal, but alternatively tumor response may be evaluated. After the operation, imatinib should be continued in the adjuvant setting for at least 3 years.

For patients with metastatic disease, imatinib is the standard treatment, with the only limiting factor being the occurrence of secondary resistance. To date, no data are available to confirm a possible adjuvant role for cytoreductive surgery to prolong imatinib activity, though further study is necessary; at present, such extensive surgery may be performed at centers with extensive experience. Salvage surgery could be considered once imatinib starts failing to a limited degree.

\section{REFERENCES}

1. Wang D, Zhang Q, Blanke CD, Demetri GD, Heinrich MC, Watson JC, et al. Phase II trial of neoadjuvant/adjuvant imatinib mesylate for advanced primary and metastatic/recurrent operable gastrointestinal stromal tumors: long-term follow-up results of radiation therapy oncology group 0132. Ann Surg Oncol. 2011. doi:10.1245/s10434-011-2190-5.

2. Demetri GD, von Mehren M, Antonescu CR, DeMatteo RP, Ganjoo KN, Maki RG, et al. NCCN Task Force report: update on the management of patients with gastrointestinal stromal tumors. J Natl Compr Cancer Netw. 2010;8(Suppl 2):S1-41.

3. Casali PG, Blay JY; ESMO/CONTICANET/EUROBONET Consensus Panel of Experts. Gastrointestinal stromal tumours: ESMO Clinical Practice Guidelines for diagnosis, treatment and follow-up. Ann Oncol. 2010;21(Suppl 5):v98-102.

4. Joensuu H, Eriksson M, Hartmann J, Sundby Hall K, Schütte J, Reichardt A, et al. Twelve versus 36 months of adjuvant imatinib (IM) as treatment of operable GIST with a high risk of recurrence: final results of a randomized trial (SSGXVIII/AIO). J Clin Oncol. 2011;15(Suppl, May 20):LBA1.

5. Dematteo RP, Ballman KV, Antonescu CR, , Maki RG, Pisters PW, Demetri GD, et al. Adjuvant imatinib mesylate after resection of localised, primary gastrointestinal stromal tumour: a randomised, double-blind, placebo-controlled trial. Lancet. 2009;373:1097-104.

6. Corless CL, Ballman BV, Antonescu C, Blanke CD, Blackstein ME, Demetri GD, et al. Relation of tumor pathologic and molecular features to outcome after surgical resection of localized primary gastrointestinal stromal tumor (GIST): results of the intergroup phase III trial ACOSOG Z9001. J Clin Oncol. 2010;28(Suppl, May 20):10006.

7. Gastrointestinal Stromal Tumor Meta-Analysis Group (MetaGIST). Comparison of two doses of imatinib for the treatment of unresectable or metastatic gastrointestinal stromal tumors: a meta-analysis of 1,640 patients. J Clin Oncol. 2010;28:1247-53.

8. Debiec-Rychter M, Sciot R, Le Cesne A, Schlemmer M, Hohenberger P, van Oosterom AT, et al. KIT mutations and dose selection for imatinib in patients with advanced gastrointestinal stromal tumours. Eur J Cancer. 2006;42:1093-103.

9. Emile JF, Brahimi S, Coindre JM, Bringuier PP, Monges G, Samb P, et al. Frequencies of KIT and PDGFRA mutations in the MolecGIST prospective population-based study differ from those of advanced GISTs. Med Oncol. 2011. doi:10.1007/s12032-011-0074-y.

10. Lassau N, Lamuraglia M, Chami L, Leclere J, Bonvalot S, Terrier $\mathrm{P}$, et al. Gastrointestinal stromal tumors treated with imatinib: monitoring response with contrast-enhanced sonography. AJR Am J Roentgenol. 2006;187:1267-73.

11. Fiore M, Palassini E, Fumagalli E, Pilotti S, Tamborini E, Stacchiotti S, et al. Preoperative imatinib mesylate for unresectable or locally advanced primary gastrointestinal stromal tumors (GIST). Eur J Surg Oncol. 2009;35:739-45.

12. Haller F, Detken S, Schulten HJ, Happel N, Gunawan B, Kuhlgatz J, et al. Surgical management after neoadjuvant imatinib therapy in gastrointestinal stromal tumours (GISTs) with 
respect to imatinib resistance caused by secondary KIT mutations. Ann Surg Oncol. 2007;14:526-32.

13. Bonvalot S, Eldweny H, Péchoux CL, Vanel D, Terrier P, Cavalcanti A, et al. Impact of surgery on advanced gastrointestinal stromal tumors (GIST) in the imatinib era. Ann Surg Oncol. 2006;13:1596-603.

14. Rutkowski P, Nowecki Z, Nyckowski P, Dziewirski W, Grzesiakowska U, Nasierowska-Guttmejer A, et al. Surgical treatment of patients with initially inoperable and/or metastatic gastrointestinal stromal tumors (GIST) during therapy with imatinib mesylate. J Surg Oncol. 2006;93:304-11.

15. Raut CP, Posner M, Desai J, Morgan JA, George S, Zahrieh D, et al. Surgical management of advanced gastrointestinal stromal tumors after treatment with targeted systemic therapy using kinase inhibitors. J Clin Oncol. 2006;24:2325-31.

16. DeMatteo RP, Maki RG, Singer S, Gonen M, Brennan MF, Antonescu CR. Results of tyrosine kinase inhibitor therapy followed by surgical resection for metastatic gastrointestinal stromal tumor. Ann Surg. 2007;245:347-52.
17. Gronchi A, Fiore M, Miselli F, Lagonigro MS, Coco P, Messina A, et al. Surgery of residual disease following molecular-targeted therapy with imatinib mesylate in advanced/metastatic GIST. Ann Surg. 2007;245:341-6.

18. Sym SJ, Ryu MH, Lee JL, Chang HM, Kim TW, Kim HC, et al. Surgical intervention following imatinib treatment in patients with advanced gastrointestinal stromal tumors (GISTs). J Surg Oncol. 2008;98:27-33.

19. Mussi C, Ronellenfitsch U, Jakob J, Tamborini E, Reichardt P, Casali PG, et al. Post-imatinib surgery in advanced/metastatic GIST: is it worthwhile in all patients? Ann Oncol. 2010;21:403-8.

20. Le Cesne A, Ray-Coquard I, Bui BN, Adenis A, Rios M, Bertucci $\mathrm{F}$, et al. Discontinuation of imatinib in patients with advanced gastrointestinal stromal tumours after 3 years of treatment: an open-label multicentre randomised phase 3 trial. Lancet Oncol. 2010;11:942-9. 